\title{
Biogenesis of Lipoproteins in Gram-Negative Bacteria: 50 Years of Progress
}

James C. Kuldell** (jkuldell||@pride.hofstra.edu)

Harshani Luknauth* (hluknauthl回pride.hofstra.edu)

Anthony E. Ricigliano* (ariciglianol国pride.hofstra.edu)

Nathan W. Rigel† (nathan.w.rigel包hofstra.edu)

Department of Biology, Hofstra University

*All authors contributed equally

$\dagger$ Corresponding Author

Manuscript received 31 January 2020; accepted 20 November 2020.

Department of Biology Hofstra University 318A Gittlesan Hall Hempstead, NY II54马 nathan.w.rigel@hofstra.edu Phone: 516-463-6542

Fax: 516-463-5112

Keywords: Acylation, Pre-prolipoprotein, Prolipoprotein, Apo-lipoprotein, Cell envelope 


\section{Abstract}

The outer membrane is the defining characteristic of Gram-negative bacteria and is crucial for the maintenance of cellular integrity. Lipoproteins are an essential component of this outer membrane and regulate broad cellular functions ranging from efflux, cellular physiology, antibiotic resistance, and pathogenicity. In the canonical model of lipoprotein biogenesis, lipoprotein precursors are first synthesized in the cytoplasm prior to extensive modifications by the consecutive action of three key enzymes: diacylglyceryl transferase $(L g t)$, lipoprotein signal peptidase A (LspA), and apolipoprotein N-acyltransferase (Lnt). This enzymatic process modifies lipoprotein precursors for subsequent trafficking by the Lol pathway. The function of these three enzymes were originally thought to be essential, however, in some Gram-negative bacteria, namely Acinetobacter baylyi, the third enzyme Lnt is dispensable. Here we review the function and significance of $L g t$, LspA, and Lnt in outer membrane biogenesis and how noncanonical models of lipoprotein processing in Acinetobacter spp. can enhance our understanding of lipoprotein modifications and trafficking.

10 | Fine Focus 


\section{Introduction}

The general structure of Gram-negative bacteria such as Escherichia coli and Acinetobacter baylyi consists of the inner membrane (IM) and outer membrane (OM) separated by an aqueous periplasm containing a thin peptidoglycan layer (1). The outer membrane is the defining characteristic of Gram-negative bacteria and is essential for the maintenance of cellular integrity. The OM is composed of phospholipids and a lipopolysaccharide asymmetric bilayer containing $\beta$-barrel OM proteins (OMPs) (2). In order for correct biogenesis of the $\mathrm{OM}$ to occur, lipopolysaccharides must be delivered to the OM by the Lpt machinery while the Beta-barrel assembly machinery (BAM) complex assembles OMPs into the OM (3). Proteins destined for the OM are synthesized in the cytoplasm with a signal peptide used for translocation to the IM by the general secretion (Sec) pathway for unfolded proteins or through the twin-arginine translocation (Tat) pathway for fully folded proteins (4-6). Deviations or loss of function in the assembly machinery needed to synthesize the $\mathrm{OM}$ results in profound defects causing morphological defects such as antibiotic and temperature sensitivity (7).

Outer membrane lipoproteins have vital functions including aiding in lipopolysaccharide (LPS) insertion in the OM and forming secretion systems $(8,9)$. LPS is found in the outer leaflet of the OM in most Gram-negative bacteria and is thus essential because it interacts directly with the outside environment of the bacterium and maintains the impermeability of the OM (10-13).

The proteins, $\mathrm{LptD}$ and $\mathrm{LptE}$ are components of the Lpt machinery which function to transport the essential LPS to the $\mathrm{OM}(8,9)$. LptE and $\mathrm{LptD}$ form a complex in which $\mathrm{LptE}$ is buried inside $\mathrm{LptD}$ and functions as a plug to prevent the passage of molecules through $\mathrm{LptD}$ into the bacteria (14-18). There are also surface-exposed lipoproteins such as Lpp and Pal which function to increase cell wall stability by attaching the OM to the cell wall (19-21). Other surfaceexposed lipoproteins are part of stress responses including RcsF which is the sensory component of the Rcs envelope stress response $(22,23)$. This stress response can be activated by LPS stress and osmotic stress (24-27).
Lipoproteins are also essential in assembly of the OM and regulating the traffic of molecules inside and outside of the Gram-negative cell (28). These OM lipoproteins can release virulence factors to the surrounding environment resulting in infection $(29,30)$. They also can release toxins or hazardous chemicals for defense (31). Another assembly of OM proteins is the BAM complex which functions to facilitate the formation of beta- barrels in the OM (32). Blocking OM lipoprotein trafficking or inhibiting their function can result in cell death (33). If a lipoprotein is not trafficked, the cell cannot regulate the efflux of molecules across the membrane, depriving it of resources and preventing the cell from expelling hazardous chemicals. Therefore, the lipoprotein sorting pathway is integral to Gram-negative bacteria because lipoproteins in the OM are essential to maintaining the cell envelope.

\section{Synthesis of lipoproteins begins in the cytoplasm.}

Lipoproteins are synthesized as precursor pre-prolipoproteins in the cytoplasm prior to being trafficked through the $\mathrm{ABC}$ transporter localization of lipoproteins (Lol) pathway (34). Once unmodified pre- lipoproteins destined for the OM are translated, they enter the IM through the Sec or Tat pathway (35) (Fig. 1a).

The signal peptide embeds the protein to the IM and allows for recognition for the first step of modification (36). Diacylglyceryl transferase $(L g t)$ is the first protein in the pathway and transfers di-acylglycerol to the cysteine residue in the lipobox of the prolipoprotein (37). Lgt has been thought to be essential in all Gram-negative bacteria because it is the first step of lipoprotein maturation which is required to be recognized and translocated using the Lol system $(37,38)$. However, insertion mutations have been created in the lgt gene of Acinetobacter baumannii which have yielded viable cultures (39). The second step of lipoprotein maturation is done by prolipoprotein signal peptidase (LspA) which cleaves the signal peptide leaving the lipobox at the $\mathrm{N}$-terminus. LspA is also essential due to its ability to cleave the anchor peptide from the IM. The heavily hydrophobic region is what allows and maintains the protein in the IM, therefore its release is integral for lipoprotein movement to the OM as well as function and folding. The third and final step in lipoprotein maturation is the addition of one more acyl Volume Seven | 11 
group to the cysteine residue by apolipoprotein transacetylase (Lnt) (Fig. 1a). Together, these processes define the canonical lipoprotein modification pathway in Gram-negative bacteria (34).

Classically it was thought that Lnt-mediated tri-acylation was an essential requirement for recognition by the Lol system and subsequent lipoprotein trafficking. However, Lnt was found to be nonessential in some Gram-negative bacteria including Francisella, Neisseria, and Acinetobacter $(40,41)$. The loss of Lnt is not without detriment, as the absence of a third acylation interrupts lipoprotein trafficking resulting in visible growth defects such as increased OM permeability in Acinetobacter spp. (42). The viability of Acinetobacter spp. after $\ln t$ deletion suggests a non-canonical function of the existing lipoprotein modification and trafficking pathway (Fig. 1b).

If an apo-lipoprotein is successfully tri-acylated and cleaved, to become a mature lipoprotein and reach the OM, it must be translocated there by the Lol system (34). The LolCDE proteins exist in the IM where it receives the tri-acylated lipoproteins, as studied in E. coli (43). If destined for the OM the periplasmic chaperone LolA then shuttles the lipoproteins to the OM receptor LolB for inclusion into the OM (38, 44-46). In Francisella spp., a fusion protein resembling E. coli LolC/LolE exists that shares high homology among functional domains of the transport system (41). This high homology of the fusion protein and subsequent pathway probing identified this protein as LolF, a distinct protein among certain gram- negative bacteria namely Neisseria, Francisella, and Acinetobacter $(42,47)$. LolF was shown to accept di-acylated lipoproteins as compared to the rejection of di-acylated lipoproteins by LolCDE (41).

The LolF arrangement and successful trafficking of di-acylated lipoproteins in Lnt-deficient $A$. baylyi suggests the presence of a non-canonical function of the lipoprotein modification and trafficking pathway (Fig. 1b). In the process of exploring the non-canonical function of this pathway, it is essential to review the known structures, functions, and mechanisms of the canonical pathway. Here we compile research spanning 50 years for the creation of a concise and accessible resource

\section{Figure 1.}

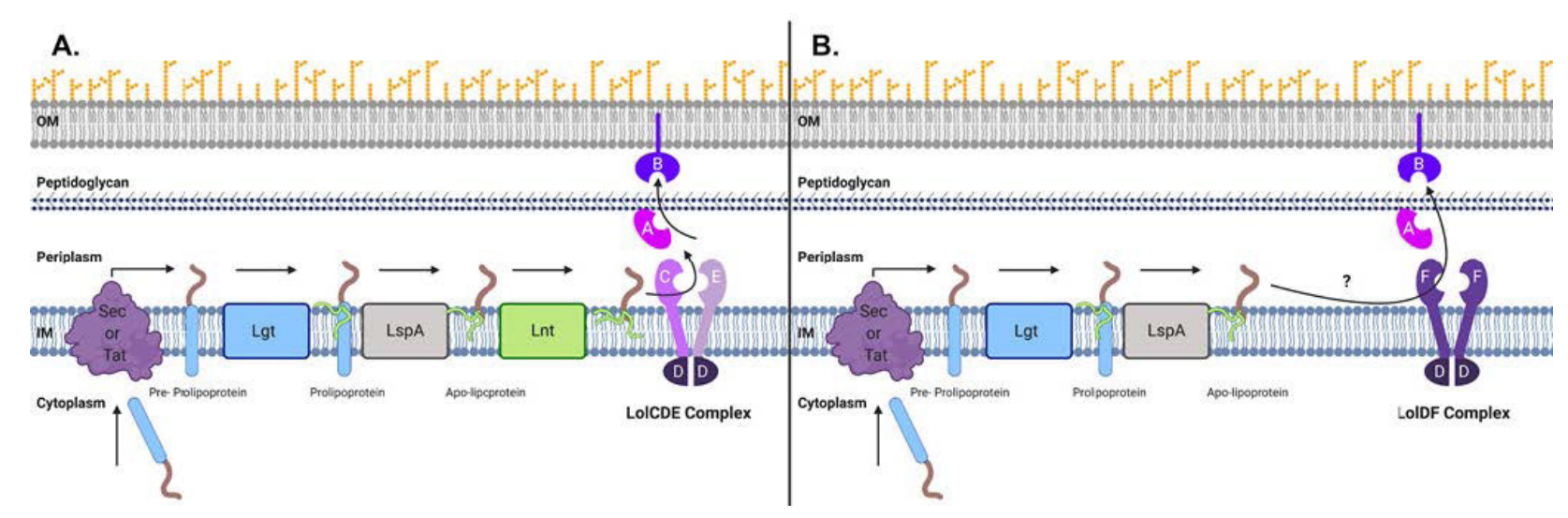

Figure 1. Lipoprotein biogenesis and trafficking in Gram-negative bacteria. (A) In the canonical lipoprotein biogenesis and trafficking pathway, pre-prolipoproteins from the cytoplasm are transported to the periplasm by the Sec or Tat pathway where Lgt adds two acyl chains (green) to the conserved cysteine residue of the lipoprotein precursor. Here, LspA cleaves the signal sequence (blue) and the third and final acyl chain is added to the amino terminus by Lnt. The IM bound LolCDE proteins then receive tri-acylated lipoproteins and can be transported by the periplasmic chaperone LolA to shuttle the lipoprotein to the OM receptor, LolB, for inclusion into the OM. (B) In the non-canonical lipoprotein trafficking pathway of Gram-negative bacteria with LolDF fusion proteins, di-acylated lipoproteins are still capable of transportation to the OM by an unknown mechanism or alternative pathway. Created with Biorender.com. 
regarding lipoprotein processing and associated enzymes within Gram-negative bacteria.

\section{Diacylglyceryl Transferase $(L g t)$.}

Before lipoproteins are translocated to the OM through the Lol system, they are first modified by Lgt in the IM (37). This IM protein di-acylates the prolipoprotein which is the first step to becoming a mature lipoprotein. All lipoproteins that have been identified have $\mathrm{N}$-acyldiacylglyceryl-cysteine as their $\mathrm{N}$-terminal amino acid (48). The lgt gene was first discovered in temperature sensitive mutants of $S$. typhimurium in which there was an accumulation of unmodified prolipoproteins at $42^{\circ} \mathrm{C}(49)$. The prolipoproteins in these temperature sensitive mutants did not have any glyceryl modification. Furthermore, a complementation test with a cloned insert of this deletion restored glyceryl modification activity. This cloned gene was determined to code for diacylglyceryl transferase $(L g t)$. In $E$. coli, the $u m p A$ gene was determined to code for $L g t$ after demonstrating high levels of similarity to the lgt gene in S. typhimurium (50).

\section{$L g t$ is generally essential in Gram-negative bacteria} when compared to Gram-positive bacteria. Lipoprotein modification has been studied more extensively in Gramnegative bacteria compared to Gram-positive bacteria. It has been determined that Gram-negative bacteria in which Lgt is essential include E. coli and S. typhimurium (49, 51, 52). However, there are some Gram-negative bacteria such as $A$. baumannii in which insertion mutations are present due to transposons in the lgt gene, yet there viable cells persist (39). Furthermore, $L g t$ has been determined to not be essential in Gram-positive organisms such as Bacillus subtilis and Streptococcus pneumoniae $(53,54)$. B. subtilis and $S$. pneumoniae cultures were still viable after $\lg t$ deletion (53). However, $\lg t$ was deemed essential for virulence in $S$. pneumoniae (54). It has been hypothesized that the function of Lgt in Gram-positive bacteria may be analogous to substrate-specific sorting enzymes which translocate wallanchored proteins $(55,56)$.

\section{Lgt consists of 291 amino acids and is comprised of} seven transmembrane helices. The crystal structure of Lgt was identified in E. coli where it is composed of 291 amino acids (33 kDa) (57). There are seven transmembrane helices which form the core of the protein and there are two phosphatidylglycerol binding sites, R143 and R239 (Fig. 2a). These two binding sites were determined to be critical through the use of complementation tests with lgt knockout cells and different mutant variants. There are six beta strands and four short helices. One of the critical catalytic sites, R143, which faces towards the periplasm, directly binds the substrate phosphatidylglycerol for transfer. This site is positively charged which makes it a prime residue for binding the negatively charged phosphatidylglycerol. The other phosphatidylglycerol binding site is E151. The residue R239 was also determined to be essential for diacylglyceryl transfer and functions by forming a hydrogen bond with the $\mathrm{C} 3$ of diacylglycerol. These two key residues are the catalytic sites which transfer di-acylglyceryl to the pre-prolipoprotein.

\section{Diacylation of the prolipoprotein begins by transferring a non-acylated glyceryl of phosphatidylglycerol to the sulfhydryl group followed by $\mathbf{O}$-acyltransferase catalyzed acylation of the glyceryl moiety. To determine} the mechanism of action for this diacyl modification, Braun's lipoprotein was used in E. coli $(25,27,28)$. Braun's lipoprotein is a murein lipoprotein which has the same composition as previously identified lipoproteins with a glyceryl cysteine (S-propane-2',3'-diol)-3-thio-2aminopropanic acid) at the peptide end attached to two fatty acids with another fatty acid bound at the N-terminal $(48,58)$. To become a mature lipoprotein, the first step of modification is done by Lgt which di-acylates the prolipoprotein (37). The mechanism of di-acylating the prolipoprotein was proposed to begin by transferring a nonacylated glyceryl of phosphatidylglycerol to the sulfhydryl group of the cysteine residue at the $\mathrm{N}$-terminal of the prolipoprotein $(48,60)$. The sn-2 and sn-3 hydroxyls of the glyceryl moiety are then acylated by $\mathrm{O}$-acyltransferase enzymes to form the di-acylglycerylated lipoprotein.

\section{Lipoprotein Signal Peptidase (LspA)}

Signal II peptidase encoded by $\operatorname{lsp} A$ in Gram-negative bacteria cleaves the signal peptide from the pre-prolipoprotein at the lipobox residue consensus sequence LAGC (L-3A-2G-1C+1) during the intermediate step of prolipoprotein processing Volume Seven | 13 
(61). The cleavage event results in an invariable Cys residue becoming the $\mathrm{N}$-terminal +1 residue, allowing for the prolipoprotein to proceed to the final acylation step by $\operatorname{Lnt}(62)$.

\section{LspA is ubiquitous among all known Gram-negative} bacteria and homologs have been found in select Grampositive bacteria. LspA is ubiquitously conserved among all known Gram-negative bacteria and homologs have been found in select Gram-positive bacteria (e.g. Staphylococcus aureus) (63). Structurally, among both Gram-positive and Gram-negative eubacteria LspA contains one conserved residue essential for stability, Asp-14, and five conserved residues important for catalytic function; Asn- 99, Asp-102, Asn-126, Ala-128, and Asp- 129 (64) (Fig. 2b). Functionally, in Gram-negative bacteria LspA is considered essential under standard laboratory conditions while in Grampositive bacteria LspA is considered conditionally essential for virulence $(65,66)$. There are no known homologs in the domain Eukaryota (63).

\section{LspA consists of 169 amino acids and is comprised of two main domains containing four transmembrane}

helices. From the crystal structure of LspA isolated from Pseudomonas aeruginosa, the 169 amino acid long (18 kDa) inner membrane protein is comprised of two main domains (67). The first domain consists of four transmembrane helices culminating in $\mathrm{N}$ and $\mathrm{C}$ termini located in the cytoplasm (68) and the second domain consists of a periplasmic domain further separated into two subdomains (67) (Fig. 2b). The larger subdomain is a $\beta$-cradle that rests on the membrane extending away from the protein's helical core presenting its polar surface to the periplasm. The smaller and second subdomain contains a periplasmic helix which extends perpendicularly from the $\beta$-cradle into the periplasm (67).

Figure 2.

A.

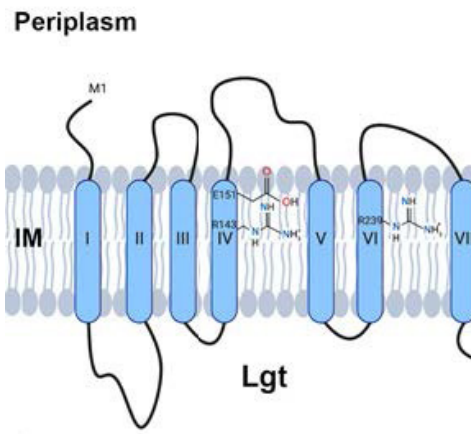

B.

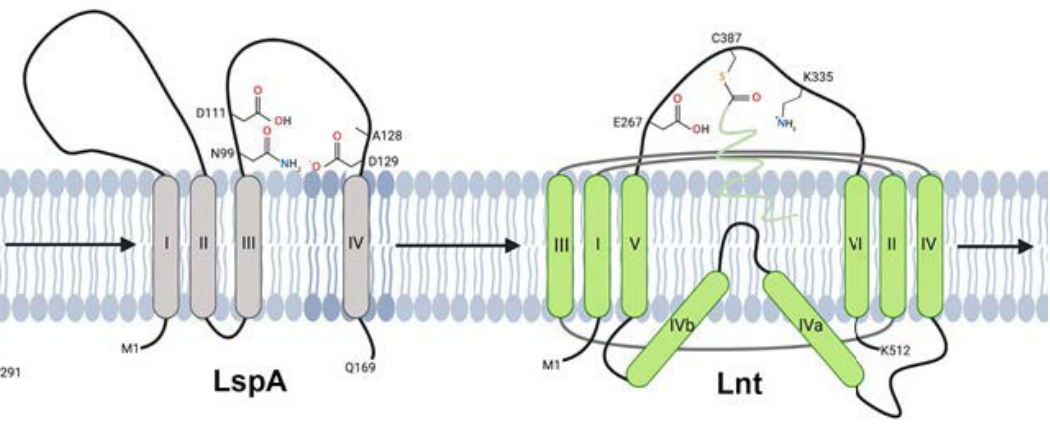

Figure 2. Proposed model of peptide topology for Lgt (blue), LspA (grey), and Lnt (green) in the inner membrane. Roman numerals indicate transmembrane domains. (A) Lgt contains seven transmembrane domains with six beta strands. PG molecules are bound to the Arg143 and Arg239 residues in transmembrane domains IV and VI respectively, with Glu151 in transmembrane IV essential to the acyl transfer. The loop between transmembrane VI and VII is a gate for the entrance of the pre-prolipoprotein. After the acyl transfer, another PG molecule docks on the protein and the process repeats. Adapted from Pailler et al., 2012. (B) LspA in addition to the transmembrane domains, has a $\beta$-cradle in the first periplasmic exposed domain that retains the majority of the lipoprotein during proteolytic cleavage. LspA also contains a periplasmic helix in the second periplasmic domain. The signal peptide of the prolipoprotein is wedged between transmembrane II, III, and IV from recognition sites Asp111, Asp129, and Asn99. Adapted from Muñoa et al., 1991 and Tjalsma et al., 1999. (C) Lnt exists as a thioester acyl intermediate with acyl group attached to C387. Lnt has a beta-barrel-like structure with a catalytic cavity. The apolipoprotein enters the cavity laterally, stabilized by the loop between transmembrane domains IVa and IVb. Adapted from Gélis- Jeanvoine et al., 2015. Created with Biorender.com. 


\section{LspA shares a mechanism of action similar to that of the}

aspartic protease family. Based on structural context and the functional domain homology to the family of aspartic proteases, LspA is proposed to form a catalytic dyad at residues Asp-102 and Asp-129 while residues Asn-45, Asn99, Asp-111, Asn-126, and Ala-128 create the geometry and recognition site for the lipobox of prolipoproteins (64). The proposed mechanism of LspA mediated signal peptide cleavage is as follows. Upon binding to a lipid-modified precursor, the carbonyl carbon of the scissile peptide bond is hydrated creating a tetrahedral intermediate (64). At this point a proton is transferred by means of a lytic water molecule on the initial protonated aspartic acid residue to another aspartic acid residue. The tetrahedral intermediate then donates a proton from one hydroxyl group to the recently charged aspartic acid residue (64). Simultaneously the nitrogen atom of the scissile peptide bond receives a proton from the catalytic aspartic acid residue resulting in the peptide bond cleavage of the signal peptide from the lipobox motif of the prolipoprotein. This proposed mechanism of LspA enzymatic action is further supported by LspA ability to function in the absence of metal ions suggesting LspA does not use classical catalytic mechanisms of metalloproteases (69).

\section{LspA presents novel targets for drug intervention of} bacterial infection. LspA is of particular significance to the development of antibiotics in response to the increasing epidemic of antibiotic resistant bacteria (70). Since LspA is broadly conserved and essential in Gram-negative bacteria and some Gram-positive bacteria it presents as a suitable target for broad-spectrum antibiotic development (63). Additionally the correct synthesis of lipoproteins by LspA-mediated enzymatic processes has been implicated in pathogenicity even in bacteria where LspA is not considered essential (e.g. Mycobacterium tuberculosis) (71). LspA is also absent in all eukaryotic cells avoiding the possibility of off-target effects on host organisms (63). In comparison to the chronological development of other antibiotics and their respective targets, LspA as a target for antibiotic development is relatively new decreasing the risk associated with "legacy" antibiotics and acquired resistance (70).
In early antibiotic discovery trials, a cyclic peptide antibiotic Globomycin was isolated from Streptomyces spp. and administered to a panel of bacteria to determine antibiotic sensitivities (72). In E. coli and other Gram-negative bacteria, growth was severely inhibited by this molecule and resulted in the formation of spheroplasts indicating profound membrane assembly defects (72). Further studies in E. coli showed Globomycin treatment resulted in the bioaccumulation of di-acylated prolipoproteins in the cytoplasmic membrane and subsequent death of the affected bacterial cell (73). In vitro incubation of LspA enzyme and prolipoprotein substrates in the presence of Globomycin showed an inhibition of LspA enzymatic activity on the cleavage of the signal peptide present on the prolipoprotein (74). Decades later, the mechanism by which Globomycin prevented LspA enzymatic activity was determined by the crystal structure of Globomycin bound to LspA from P. aeruginosa (67). Globomycin was found to infiltrate the LspA binding pocket consisting of conserved residues, typically specific for prolipoprotein substrates, and tightly bind both aspartic residues implicated in cleavage as described above (67). The occupation of both active enzymatic residues prevents typical enzymatic function resulting in the observed OM defects and subsequent death of the bacterium. Globomycin is an efficient inhibitor of Gram-negative bacterial growth and affords the benefit of targeting LspA, which has no eukaryotic homolog. Therefore, the development of Globomycin analogs which can more efficiently mimic prolipoproteins and bind LspA is considered a promising avenue of research in the development of new antimicrobials (67).

Another bacterial secondary metabolite derived antibiotic, myxovirescin, was found to inhibit Gram-negative bacteria prolipoprotein processing and subsequent growth in a mechanism similar to that of Globomycin despite having a unique molecular structure (75). Even though operating on convergent mechanisms of LspA inhibition, myxovirescin was shown to be rapidly bactericidal by a magnitude of almost 10-fold compared to Globomycin (75). The advent of another LspA targeted antibiotic with even less incidence of spontaneous resistance compared to Globomycin reinforces the importance of LspA as a viable target for antibiotic discovery (63). 


\section{Apolipoprotein N-acyltransferase (Lnt)}

The last post-translational modification in the lipoprotein sorting pathway is performed by apolipoprotein $\mathrm{N}$-acyltransferase (Lnt), an IM bound protein. Lnt acquires an acyl group from a glycerophospholipid and transfers to the +1 cysteine, the same residue previously bonded to the now cleaved signal peptide, via an amide bond $(52,76)$. This final modification acts as a conformational structure for Lol system translocation to the OM (77).

\section{Lnt is conserved among Gram-negative bacteria but has} been found in Mycobacterium spp. Lnt is extremely well conserved among Gram-negative bacteria but has also been found in Mycobacterium $(78,79)$. While Lgt and LspA are universally prominent among both Gram-negative and Gram-positive bacteria, Lnt is not. The purpose of the third acylation by Lnt is thought to be for recognition for translocation to the OM, therefore Lnt does not have a function in Gram-positives. Interestingly, Lnt homologs have been identified from BLASTp in Mycobacterium smegmatis and Mycobacterium tuberculosis as Ppm1. Ppm1 has also been confirmed to transfer an acetyl group to the $\mathrm{N}$-terminus, similar to the Lnt mechanism (78). The tri-acylated lipoproteins are suspected to contribute to $M$. tuberculosis virulence factors (71).

\section{Glycerophospholipids are the prominent substrates}

for Lnt. Lnt transfers an acyl group from 3 different glycerophospholipids. Phosphatidylethanolamine (PE), the most abundant phospholipid, constituting around $70 \%$ of the cellular lipid content, is the prominent substrate for Lnt, but not essential for Lnt function (80). Phosphatidylglycerol (PG), the second most abundant phospholipid, is a substrate for Lnt as well, but not used as efficiently as $\operatorname{PE}(81,82)$. Interestingly, in mutants lacking PE, PG was used for final acylation without defects. Phosphatidic acids (PA) is one of the least abundant membrane lipids but can be used as a substitute for PG efficiently.

\section{Lnt consists of 512 amino acids and is comprised of six} transmembrane segments. Lnt has six transmembrane (TMS) segments with both the carboxy-terminus and

\section{佰 | Fine Focus}

amino-terminus exposed towards the cytoplasm (83). A large, 79 amino acid long, cytoplasmic loop (CL-2) is present between TMS-IV and TMS-V and contains two hydrophobic segments (TMS-IVa and TMS-IVb) (83). These hydrophobic segments are not fully integrated into the membrane and are partially exposed to the cytoplasm (Fig. 2c). Similar morphology to a reentrant loop motif, these segments may be used for channeling in substrates, but CL-2 lacks key secondary structure for a reentrant loop (84). It is hypothesized that instead, CL-2 forms a titled fold similar to an intramembrane protease GlpG in E. coli $(85,86)$. Lnt also contains a periplasmic exposed nitrilase domain between TSM-V and TSM-VI (83). It is proposed that CL-2, TSM$\mathrm{IVa}$, and TSM-IVb are surrounded by the six TMS forming a beta-barrel-like structure (85). Lnt contains a catalytic cavity inside the beta-barrel-like structure, but the lipoprotein substrate enters the structure laterally, a mechanism similar to integral membrane proteins (87), Lnt also exists as a thioester acyl intermediate to allow for higher processivity and acylation for essential OM-bound lipoproteins (52).

The mechanism for acylation transfer is a two-step process comprised of auto-acylation followed by acyl transfer. The mechanism for acylation transfer is most likely a two-step process, auto-acylation followed by acyl transfer $(52,81)$. Due to the massive amount of lipoproteins transported to the $\mathrm{OM}$ and the low abundance of the enzyme, Lnt existing as an acylated intermediate allows for higher processivity of the acyl transfer $(52,81)$. The active site of Lnt is in the nitrilase domain exposed to the periplasm. For auto-acylation, C387 sulfhydryl group initiates with a nucleophilic attack on the alpha carbonyl of a phospholipid. The resonance from E267 attacks the hydrogen on C387 sulfhydryl group allowing the fatty acid chain to remain on C387, yielding a thioester acyl Lnt (52). For the acyl transfer, the newly exposed nitrogen on the di-acylated lipoprotein then attacks the alpha-carbon of the acyl group on C387. The next step requires C387, K335, and E267 on the nitrilase domain, W237 on the $\beta 1 / \alpha 1$ loop, F358 and M362 on $\beta 5 / \beta 6$ loop, and R139 and P147 on CL-2 (85).

Lnt is essential in E. coli containing the LolCDE pathways, but not other Gram-negative bacteria which have LolFD pathways. In E. coli, $\ln t$ is essential. Without the final acyl 
modification, OM destined lipoproteins are mislocalized (77). This results in envelope stress and an inability to produce functional channel proteins (73). However, in Acinetobacter baylyi, Acinetobacter baumannii, Francisella tularensis, and Neisseria gonorrhoeae, $\ln t$ is not essential (88). It is unclear if these Gram-negative bacteria can translocate di-acylated lipoproteins or another enzyme performs the same function. Francisella and Acinetobacter produce a novel Lol complex, LolDF, as opposed to the LolCDE characterized in E. coli and many other Gram-negative bacteria (41) (Fig. 1b). In transcriptomic studies of Lnt deficient $A$. baylyi, LolA is upregulated significantly (88) but it is improbable that this upregulation can solely account for $A$. baylyi survival in Lnt deficient states. Data from the same transcriptome set shows a twenty-fold increase in $h s l$, gene expression in Lnt deficient A. baylyi (88). Additionally, a crystal structures of E. coli LolA (89) and putative structure of E. coli HslJ (NP_415897.1, EMBL-EBI) are very similar in structure. Due to the highly specific mouth-to-mouth transfer mechanism (90) that LolA and LolB interact with one another to transfer their inner lipoprotein cargo, homology in structure among LolA and $H s l J$ is a reasonable cause to investigate $\mathrm{HslJ}$ as a potential chaperone suppressing the deleterious effects of

Lnt deficiency.

\section{Concluding Remarks}

The lipoprotein sorting pathway is more complex than initially thought, the complexity and conditionally essential nature of the genes involved in the processing and transportation pathways provides opportunities to develop additional antimicrobial compounds for both clinical and small molecule pathway-probing applications. Though it was initially assumed that the action of $L g t$, LspA, and Lnt were needed for lipoprotein biogenesis in all Gram-negative bacteria, this now seems to be an over-simplification. It is certainly true that most Gram-negatives require functional Lgt, LspA, and Lnt to be viable $(50,52,83)$. However, it is now clear that there are many exceptions to this rule. $A$. baylyi is viable without $\mathrm{Lnt}$ and requires no other genetic manipulations to grow (88). In this species, the potentially more promiscuous LolCDE analog LolDF is used and a di-acylated lipoprotein is able to be recognized for transport instead of one that is tri-acylated by a mechanism that is still unknown (Fig. 1b) (88). By exploiting the ability of $A$. baylyi to survive in Lnt-deficient cellular environments we can use A. baylyi as a model to explore novel lipoprotein processing and transportation constituents. Uncovering the mechanism of which $A$. baylyi can overcome Lnt deficiency is essential and in doing so, we open new avenues of non-canonical OM biogenesis pathways and chemical interventions.

\section{Acknowledgements}

This work was supported by the National Science Foundation under Grant No. 1615822 to NWR 


\section{Glossary of Terms}

$\boldsymbol{\beta}$-barrel assembly machine (BAM): Five-protein complex that assembles $\beta$-barrel proteins into the outer membrane

General secretion (Sec) pathway: System for exporting unfolded proteins from the cytoplasm into the inner membrane

Twin arginine translocation (Tat) pathway: System for exporting folded proteins from the cytoplasm into the inner membrane

Lipopolysaccharide Transport (Lpt) machinery: Transporter system to shuttle lipopolysaccharides across the periplasm to the outer membrane

Localization of lipoprotein (Lol) pathway: Pathway responsible for trafficking mature lipoproteins from the inner membrane to the outer membrane

Pre-prolipoprotein: Precursor lipoproteins exported from the cytoplasm prior to acylation by Lgt Prolipoprotein: Precursor lipoprotein with two acyl chains from the action of $L g t$ and with a still-intact signal peptide

Apo-lipoprotein: Di-acylated precursor lipoprotein with cleaved signal peptide prior to final acylation from Lnt 


\section{References}

1. Grabowicz M, Silhavy TJ. 2017. Redefining the essential trafficking pathway for outer membrane lipoproteins. Proc Natl Acad Sci 114:4769-4774.

2. Okuda S, Sherman DJ, Silhavy TJ, Ruiz N, Kahne D. 2016. Lipopolysaccharide transport and assembly at the outer membrane: the PEZ model. Nat Rev Microbiol 14:337-345.

3. Hagan CL, Silhavy TJ, Kahne D. 2011. $\beta$-Barrel membrane protein assembly by the Bam complex. Annu Rev Biochem 80:189-210.

4. Mori H, Ito K. 2001. The Sec protein-translocation pathway. Trends Microbiol 9:494-500.

5. Sargent F, Bogsch EG, Stanley NR, Wexler M, Robinson C, Berks BC, Palmer T. 1998. Overlapping functions of components of a bacterial Sec-independent protein export pathway. EMBO J 17:3640-3650.

6. The bacterial twin-arginine translocation pathway. - PubMed - NCBI.

7. Choi U, Lee C-R. 2019. Distinct Roles of Outer Membrane Porins in Antibiotic Resistance and Membrane Integrity in Escherichia coli. Front Microbiol 10:953.

8. Chng S-S, Ruiz N, Chimalakonda G, Silhavy TJ, Kahne D. 2010. Characterization of the two- protein complex in Escherichia coli responsible for lipopolysaccharide assembly at the outer membrane. Proc Natl Acad Sci US A 107:53635368.

9. Wu T, McCandlish AC, Gronenberg LS, Chng S-S, Silhavy TJ, Kahne D. 2006. Identification of a protein complex that assembles lipopolysaccharide in the outer membrane of Escherichia coli. Proc Natl Acad Sci U S A 103:11754-11759.

10. Sperandeo P, Martorana AM, Polissi A. 2017. The lipopolysaccharide transport (Lpt) machinery: A nonconventional transporter for lipopolysaccharide assembly at the outer membrane of Gram-negative bacteria. J Biol Chem 292:1798117990. 11. Kamio Y, Nikaido H. 1976. Outer membrane of Salmonella typhimurium: accessibility of phospholipid head groups to phospholipase C and cyanogen bromide activated dextran in the external medium. Biochemistry 15:25612570 .

11. Nikaido H. 2003. Molecular basis of bacterial outer membrane permeability revisited. Microbiol Mol Biol Rev MMBR 67:593-656.

12. Nikaido H. 2005. Restoring permeability barrier function to outer membrane. Chem Biol 12:507-509.

13. Qiao S, Luo Q, Zhao Y, Zhang XC, Huang Y. 2014. Structural basis for lipopolysaccharide insertion in the bacterial outer membrane. Nature 511:108-111.

14. Dong H, Xiang Q, Gu Y, Wang Z, Paterson NG, Stansfeld PJ, He C, Zhang Y, Wang W, Dong C. 2014. Structural basis for outer membrane lipopolysaccharide insertion. Nature 511:52-56.

15. Freinkman E, Chng S-S, Kahne D. 2011. The complex that inserts lipopolysaccharide into the bacterial outer membrane forms a two-protein plug-and-barrel. Proc Natl Acad Sci U S A 108:2486- 2491.

Volume Seven | 19 
16. Malojčić G, Andres D, Grabowicz M, George AH, Ruiz N, Silhavy TJ, Kahne D. 2014. LptE binds to and alters the physical state of LPS to catalyze its assembly at the cell surface. Proc Natl Acad Sci 111:9467-9472.

17. Grabowicz M, Yeh J, Silhavy TJ. 2013. Dominant Negative lptE Mutation That Supports a Rol for LptE as a Plug in the LptD Barrel. J Bacteriol 195:1327-1334.

18. Hirota Y, Suzuki H, Nishimura Y, Yasuda S. 1977. On the process of cellular division in Escherichia coli: a mutant of $E$. coli lacking a murein-lipoprotein. Proc Natl Acad Sci U S A 74:1417-1420.

19. Suzuki H, Nishimura Y, Yasuda S, Nishimura A, Yamada M, Hirota Y. 1978. Murein-lipoprotein of Escherichia coli: A protein involved in the stabilization of bacterial cell envelope. Mol Gen Genet MGG 167:1-9.

20. Lazzaroni JC, Portalier R. 1992. The excC gene of Escherichia coli K-12 required for cell envelope integrity encodes the peptidoglycan-associated lipoprotein (PAL). Mol Microbiol 6:735-742.

21. Majdalani N, Heck M, Stout V, Gottesman S. 2005. Role of RcsF in Signaling to the Rcs Phosphorelay Pathway in Escherichia coli. J Bacteriol 187:6770-6778.

22. Castanié-Cornet M-P, Cam K, Jacq A. 2006. RcsF Is an Outer Membrane Lipoprotein Involved in the RcsCDB Phosphorelay Signaling Pathway in Escherichia coli. J Bacteriol 188:4264-4270.

23. Parker CT, Kloser AW, Schnaitman CA, Stein MA, Gottesman S, Gibson BW. 1992. Role of the rfaG and rfaP genes in determining the lipopolysaccharide core structure and cell surface properties of Escherichia coli K-12. J Bacteriol 174:2525-2538.

24. Farris C, Sanowar S, Bader MW, Pfuetzner R, Miller SI. 2010. Antimicrobial peptides activate the Rcs regulon through the outer membrane lipoprotein RcsF. J Bacteriol 192:4894-4903.

25. Ophir T, Gutnick DL. 1994. A role for exopolysaccharides in the protection of microorganisms from desiccation. Appl Environ Microbiol 60:740-745.

26. Sledjeski DD, Gottesman S. 1996. Osmotic shock induction of capsule synthesis in Escherichia coli K-12. J Bacteriol 178:1204-1206.

27. Silhavy TJ, Kahne D, Walker S. 2010. The Bacterial Cell Envelope. Cold Spring Harb Perspect Biol 2: a000414.

28. Mei JM, Nourbakhsh F, Ford CW, Holden DW. 1997. Identification of Staphylococcus aureus virulence genes in a murine model of bacteremia using signature-tagged mutagenesis. Mol Microbiol 26:399-407.

29. Tidhar A, Flashner Y, Cohen S, Levi Y, Zauberman A, Gur D, Aftalion M, Elhanany E, Zvi A, Shafferman A, Mamroud E. 2009. The NlpD lipoprotein is a novel Yersinia pestis virulence factor essential for the development of plague. PloS One 4:e7023.

30. Zhuk I, Jariwala F, Attygalle AB, Wu Y, Libera MR, Sukhishvili SA. 2014. Self-Defensive Layer- by-Layer Films with Bacteria-Triggered Antibiotic Release. ACS Nano 8:7733-7745.

31. Kim KH, Aulakh S, Paetzel M. 2012. The bacterial outer membrane $\beta$-barrel assembly machinery. Protein Sci Publ Protein Soc 21:751-768. 
32. Grabowicz M. 2018. Lipoprotein Transport: Greasing the Machines of Outer Membrane Biogenesis: Re-Examining Lipoprotein Transport Mechanisms Among Diverse Gram-Negative Bacteria While Exploring New Discoveries and Questions. BioEssays News Rev Mol Cell Dev Biol 40: e1700187.

33. Okuda S, Tokuda H. 2011. Lipoprotein Sorting in Bacteria. Annu Rev Microbiol 65:239-259.

34. Narita S-I, Tokuda H. 2017. Bacterial lipoproteins; biogenesis, sorting and quality control. Biochim Biophys Acta Mol Cell Biol Lipids 1862:1414-1423.

35. Seydel A, Gounon P, Pugsley AP. 1999. Testing the " +2 rule" for lipoprotein sorting in the Escherichia coli cell envelope with a new genetic selection. Mol Microbiol 34:810-821.

36. Sankaran K, Wu HC. 1994. Lipid modification of bacterial prolipoprotein. Transfer of diacylglyceryl moiety from phosphatidylglycerol. J Biol Chem 269:19701-19706.

37. Matsuyama S, Tajima T, Tokuda H. 1995. A novel periplasmic carrier protein involved in the sorting and transport of Escherichia coli lipoproteins destined for the outer membrane. EMBO J 14:3365-3372.

38. Gallagher LA, Ramage E, Weiss EJ, Radey M, Hayden HS, Held KG, Huse HK, Zurawski DV, Brittnacher MJ, Manoil C. 2015. Resources for Genetic and Genomic Analysis of Emerging Pathogen Acinetobacter baumannii. J Bacteriol 197:2027-2035.

39. Chahales P, Thanassi DG. 2015. A more flexible lipoprotein sorting pathway. J Bacteriol 197:1702-1704.

40. LoVullo ED, Wright LF, Isabella V, Huntley JF, Pavelka MS. 2015. Revisiting the Gram-negative lipoprotein paradigm. J Bacteriol 197:1705-1715.

41. Gwin CM, Prakash N, Christian Belisario J, Haider L, Rosen ML, Martinez LR, Rigel NW. 2018. The apolipoprotein $\mathrm{N}$-acyl transferase Lnt is dispensable for growth in Acinetobacter species. Microbiol Read Engl 164:1547-1556.

42. Yakushi T, Masuda K, Narita S, Matsuyama S, Tokuda H. 2000. A new ABC transporter mediating the detachment of lipid-modified proteins from membranes. Nat Cell Biol 2:212-218.

43. Yakushi T, Yokota N, Matsuyama S, Tokuda H. 1998. LolA-dependent release of a lipid-modified protein from the inner membrane of Escherichia coli requires nucleoside triphosphate. J Biol Chem 273:32576-32581.

44. Matsuyama S i, Yokota N, Tokuda H. 1997. A novel outer membrane lipoprotein, LolB (HemM), involved in the LolA (p20)-dependent localization of lipoproteins to the outer membrane of Escherichia coli. EMBO J 16:6947-6955.

45. Narita S, Tanaka K, Matsuyama S, Tokuda H. 2002. Disruption of lolCDE, Encoding an ATP- Binding Cassette Transporter, Is Lethal for Escherichia coli and Prevents Release of Lipoproteins from the Inner Membrane. J Bacteriol 184:1417-22.

46. da Silva RAG, Churchward CP, Karlyshev AV, Eleftheriadou O, Snabaitis AK, Longman MR, Ryan A, Griffin R. 2017. The role of apolipoprotein $\mathrm{N}$-acyl transferase, Lnt, in the lipidation of factor $\mathrm{H}$ binding protein of Neisseria meningitidis strain MC58 and its potential as a drug target. Br J Pharmacol 174:2247-2260.

47. Hantke K, Braun V. 1973. Covalent binding of lipid to protein. Diglyceride and amide-linked fatty acid at the N-terminal end of the murein-lipoprotein of the Escherichia coli outer membrane. Eur J Biochem 34:284-296. 
48. Gan K, Gupta SD, Sankaran K, Schmid MB, Wu HC. 1993. Isolation and characterization of a temperature-sensitive mutant of Salmonella typhimurium defective in prolipoprotein modification. J Biol Chem 268:16544-16550.

49. Gan K, Sankaran K, Williams MG, Aldea M, Rudd KE, Kushner SR, Wu HC. 1995. The umpA gene of Escherichia coli encodes phosphatidylglycerol:prolipoprotein diacylglyceryl transferase $(g t)$ and regulates thymidylate synthase levels through translational coupling. J Bacteriol 177:1879-1882.

50. Chattopadhyay PK, Lai JS, Wu HC. 1979. Incorporation of phosphatidylglycerol into murein lipoprotein in intact cells of Salmonella typhimurium by phospholipid vesicle fusion. J Bacteriol 137:309-312.

51. Buddelmeijer N, Young R. 2010. The Essential Escherichia coli Apolipoprotein N-Acyltransferase (Lnt) Exists as an Extracytoplasmic Thioester Acyl-Enzyme Intermediate. Biochemistry 49:341-346.

52. Leskelä S, Wahlström E, Kontinen VP, Sarvas M. 1999. Lipid modification of prelipoproteins is dispensable for growth but essential for efficient protein secretion in Bacillus subtilis: characterization of the lgt gene. Mol Microbiol 31:10751085 .

53. Petit CM, Brown JR, Ingraham K, Bryant AP, Holmes DJ. 2001. Lipid modification of prelipoproteins is dispensable for growth in vitro but essential for virulence in Streptococcus pneumoniae. FEMS Microbiol Lett 200:229-233.

54. Hutchings MI, Palmer T, Harrington DJ, Sutcliffe IC. 2009. Lipoprotein biogenesis in Gram- positive bacteria: knowing when to hold 'em, knowing when to fold 'em. Trends Microbiol 17:13-21.

55. Marraffini LA, Dedent AC, Schneewind O. 2006. Sortases and the art of anchoring proteins to the envelopes of Grampositive bacteria. Microbiol Mol Biol Rev MMBR 70:192-221.

56. Mao G, Zhao Y, Kang X, Li Z, Zhang Y, Wang X, Sun F, Sankaran K, Zhang XC. 2016. Crystal structure of E. coli lipoprotein diacylglyceryl transferase. Nat Commun 7:1-12.

57. Braun V, Rehn K. 1969. Chemical Characterization, Spatial Distribution and Function of a Lipoprotein (MureinLipoprotein) of the E. coli Cell Wall. Eur J Biochem 10:426-438.

58. Hantke K, Braun V. 1973. Covalent Binding of Lipid to Protein. Eur J Biochem 34:284-296.

59. Hayashi S, Wu HC. 1990. Lipoproteins in bacteria. J Bioenerg Biomembr 22:451-471.

60. Tokunaga M, Loranger JM, Wolfe PB, Wu HC. 1982. Prolipoprotein signal peptidase in Escherichia coli is distinct from the M13 procoat protein signal peptidase. J Biol Chem 257:9922-9925.

61. Tokunaga M, Tokunaga H, Wu HC. 1982. Post-translational modification and processing of Escherichia coli prolipoprotein in vitro. Proc Natl Acad Sci 79:2255-2259.

62. Xiao Y, Wall D. 2014. Genetic redundancy, proximity, and functionality of lspA, the target of antibiotic TA, in the Myxococcus xanthus producer strain. J Bacteriol 196:1174-1183.

63. Tjalsma H, Zanen G, Venema G, Bron S, Dijl JM van. 1999. The Potential Active Site of the Lipoprotein-specific (Type II) Signal Peptidase of Bacillus subtilis. J Biol Chem 274:28191-28197.

64. Hussain M, Ichihara S, Mizushima S. 1982. Mechanism of signal peptide cleavage in the biosynthesis of the major lipoprotein of the Escherichia coli outer membrane. J Biol Chem 257:5177- 5182.

22 | Fine Focus 
65. Tjalsma H, Kontinen VP, Prágai Z, Wu H, Meima R, Venema G, Bron S, Sarvas M, Dijl JM van. 1999. The Role of Lipoprotein Processing by Signal Peptidase II in the Gram-positive Eubacterium Bacillus subtilis Signal peptidase ii is required for the efficient secretion of a-amylase, a non-lipoprotein. J Biol Chem 274:1698-1707.

66. Vogeley L, Arnaout TE, Bailey J, Stansfeld PJ, Bo land C, Caffrey M. 2016. Structural basis of lipoprotein signal peptidase II action and inhibition by the antibiotic globomycin. Science 351:876-880.

67. Muñoa FJ, Miller KW, Beers R, Graham M, Wu HC. 1991. Membrane topology of Escherichia coli prolipoprotein signal peptidase (signal peptidase II). J Biol Chem 266:17667-17672.

68. Novak P, Ray PH, Dev IK. 1986. Localization and purification of two enzymes from Escherichia coli capable of hydrolyzing a signal peptide. J Biol Chem 261:420-427.

69. Nodwell JR. 2007. Novel Links between Antibiotic Resistance and Antibiotic Production. J Bacteriol 189:3683-3685.

70. Sander P, Rezwan M, Walker B, Rampini SK, Kroppenstedt RM, Ehlers S, Keller C, Keeble JR, Hagemeier M, Colston MJ, Springer B, Böttger EC. 2004. Lipoprotein processing is required for virulence of Mycobacterium tuberculosis. Mol Microbiol 52:1543-1552.

71. Inukai M, Nakajima M, Osawa M, Haneishi T, Arai M. 1978. Globomycin, a new peptide antibiotic with spheroplastforming activity. J Antibiot (Tokyo) 31:421-425.

72. Hussain M, Ichihara S, Mizushima S. 1980. Accumulation of glyceride-containing precursor of the outer membrane lipoprotein in the cytoplasmic membrane of Escherichia coli treated with globomycin. J Biol Chem 255:3707-3712.

73. Tokunaga M, Loranger JM, Wu HC. 1983. Isolation and characterization of an Escherichia coli clone overproducing prolipoprotein signal peptidase. J Biol Chem 258:12102-12105.

74. Xiao Y, Gerth K, Müller R, Wall D. 2012. Myxobacterium-Produced Antibiotic TA (Myxovirescin) Inhibits Type II Signal Peptidase. Antimicrob Agents Chemother 56:2014-2021.

75. Gupta SD, Wu HC. 1991. Identification and subcellular localization of apolipoprotein N-acyltransferase in Escherichia coli. FEMS Microbiol Lett 62:37-41.

76. Pugsley AP. 1993. The Complete General Secretory Pathway in Gram-Negative Bacteria. Microbiol Rev 57:59.

77. Tschumi A, Nai C, Auchli Y, Hunziker P, Gehrig P, Keller P, Grau T, Sander P. 2009. Identification of Apolipoprotein N-Acyltransferase (Lnt) in Mycobacteria. J Biol Chem 284:27146-27156.

78. Brülle JK, Tschumi A, Sander P. 2013. Lipoproteins of slow-growing Mycobacteria carry three fatty acids and are N-acylated by Apolipoprotein N-Acyltransferase BCG_2070c. BMC Microbiol 13:223.

79. Gupta SD, Dowhan W, Wu HC. 1991. Phosphatidylethanolamine is not essential for the N-acylation of apolipoprotein in Escherichia coli. J Biol Chem 266:9983-9986.

80. Hillmann F, Argentini M, Buddelmeijer N. 2011. Kinetics and Phospholipid Specificity of Apolipoprotein N-Acyltransferase. J Biol Chem 286:27936-27946.

81. Schaechter M. 2001. Escherichia coli and Salmonella 2000: the View From Here. Microbiol Mol Biol Rev 65:119-130. 
82. Robichon C, Vidal-Ingigliardi D, Pugsley AP. 2005. Depletion of Apolipoprotein N-Acyltransferase Causes Mislocalization of Outer Membrane Lipoproteins in Escherichia coli. J Biol Chem 280:974-983.

83. Yan C, Luo J. 2010. An analysis of reentrant loops. Protein J 29:350-354.

84. Gélis-Jeanvoine S, Lory S, Oberto J, Buddelmeijer N. 2015. Residues located on membrane- embedded flexible loops are essential for the second step of the apolipoprotein N-acyltransferase reaction. Mol Microbiol 95:692-705.

85. Ben-Shem A, Fass D, Bibi E. 2007. Structural basis for intramembrane proteolysis by rhomboid serine proteases. Proc Natl Acad Sci U S A 104:462-466.

86. Lu G, Xu Y, Zhang K, Xiong Y, Li H, Cui L, Wang X, Lou J, Zhai Y, Sun F, Zhang XC. 2017. Crystal structure of E. coli apolipoprotein $\mathrm{N}$-acyl transferase. Nat Commun 8:1-8.

87. Gwin CM, Prakash N, Christian Belisario J, Haider L, Rosen ML, Martinez LR, Rigel NW. 2018. The apolipoprotein $\mathrm{N}$-acyl transferase Lnt is dispensable for growth in Acinetobacter species. Microbiol Read Engl 164:1547-1556.

88. Takeda K, Miyatake H, Yokota N, Matsuyama S, Tokuda H, Miki K. 2003. Crystal structures of bacterial lipoprotein localization factors, LolA and LolB. Embo J 22:3199-3209.

89. Okuda S, Tokuda H. 2009. Model of mouth-to-mouth transfer of bacterial lipoproteins through inner membrane LolC, periplasmic LolA, and outer membrane LolB. Proc Natl Acad Sci 106:5877- 5882.

90. Pailler J, Aucher W, Pires M, Buddelmeijer N. 2012. Phosphatidylglycerol::prolipoprotein diacylglyceryl transferase $(L g t)$ of Escherichia coli has seven transmembrane segments, and its essential residues are embedded in the membrane. $J$ Bacteriol 194:2142-2151. 